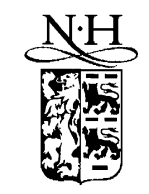

Available online at www.sciencedirect.com

ELSEVIER

Journal of Magnetism and Magnetic Materials 286 (2005) 1-4

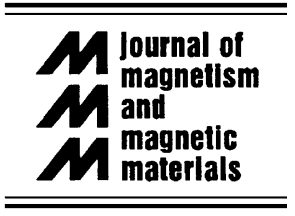

www.elsevier.com/locate/jmmm

\title{
Ordered sub-micron magnetic dot arrays using self-assembly template method
}

\author{
A. Goncharov ${ }^{\mathrm{a}, *}$, A.A. Zhukov ${ }^{\mathrm{a}}$, P.N. Bartlett ${ }^{\mathrm{b}}$, M.A. Ghanem ${ }^{\mathrm{b}}$, R. Boardman ${ }^{\mathrm{c}}$, \\ H. Fangohr ${ }^{\mathrm{c}}$, P.A.J. de Groot ${ }^{\mathrm{a}}$ \\ ${ }^{a}$ School of Physics and Astronomy, University of Southampton, Southampton, SO17 1BJ, UK \\ ${ }^{\mathrm{b}}$ School of Chemistry, University of Southampton, Southampton, SO17 1BJ, UK \\ ${ }^{\mathrm{c}}$ Computational Engineering and Design Group, School of Engineering Sciences, University of Southampton, SO17 1BJ, UK
}

Available online 8 October 2004

\begin{abstract}
Using a self-assembly double template method, we have prepared highly ordered magnetic dot arrays of Ni on submicron scales. For the template preparation we have used colloidal suspensions of polystyrene latex spheres with different diameters ranging from 200 to $700 \mathrm{~nm}$ combined with secondary templates of the conducting polymer polypyrrole. By variation of the template parameters and the deposition charge the material shape can be modified significantly from isolated dots to connected 3D networks. Samples have been characterized by magnetometry. Magnetic properties were found to depend strongly on dot size and connectivity leading to a rich variety of magnetic behaviors.

(C) 2004 Published by Elsevier B.V.
\end{abstract}

PACS: $75.50 . \mathrm{Ss}$

Keywords: Magnetic arrays; Nanomagnetics; Self-assembly; Template methods; Vortex

\section{Introduction}

Recent achievements in fabricating sub-micron magnetic patterned materials offer excellent perspectives for magnetic applications [1,2]. In particular, they hold the promise of breaking the

\footnotetext{
*Corresponding author. Tel.: + 442380592104 ; fax: +442380593910 .

E-mail address: gubbiotti@fisica.unipg.it (A. Goncharov).
}

superparamagnetic limit in data storage. However, the cost of lithographically prepared arrays becomes extremely high for small structures with lateral dimensions below $200 \mathrm{~nm}$. Developing relatively cheap methods for fabricating nanoscale structures is one of the most important issues in nano-physics today. We present results on magnetic dot arrays prepared using a novel double-template electrodeposition method. This method allowed us to grow dot arrays made of $\mathrm{Ni}$ 
with periods ranging from 200 to $700 \mathrm{~nm}$. The diameter of the dots can be set from $20 \mathrm{~nm}$. The quasi-spherical shape of dots resulting from our fabricating process has been found to be of importance for the magnetic properties.

\section{Sample preparation}

At present there are several methods used to create ordered sub-micron magnetic structures such as e-beam lithography, X-ray and interference lithography and others [3]. Recently, much attention has been directed towards template methods [4-6]. In our case the electro-deposition through hard self-assembly templates has been used for preparation of highly regular nano-scale dot arrays. The templates were prepared by selfassembly of polystyrene latex spheres on a goldcoated glass substrate. For this purpose a $0.5 \mathrm{wt} \%$ water suspension of the polystyrene spheres was spread on the substrate. Subsequently, the water was allowed to evaporate slowly for 3-5 days. During evaporation the spheres self-assemble by capillary and electrostatic forces into a 3D close packed structure. This ordered structure of spheres forms the primary template. This primary template was used for the electrodeposition of the conducting polymer polypyrrole resulting in a macroporous polymer template. After the deposition of polypyrrole the polystyrene spheres were dissolved in toluene leaving a secondary polymer template (Fig. 1). The polypyrrole was then converted into an insulator either by over-oxidation or by undoping at a sufficiently negative potential. This insulating structure was used as the template for electrochemical deposition of magnetic material. At the point where the polystyrene spheres-prior to being removed-touched the substrate, discshaped areas with a diameter of $\sim d / 4$ of gold surface are exposed. Electrodeposition of magnetic materials starts at these points gradually filling the spherical cavities of the polypyrrole template. More details about the preparation of secondary templates are given in Ref. [7]. Using this technique, we have prepared ordered arrays of dots with quasi-spherical geometry. The thickness of the dots $t$ can be controlled by varying the

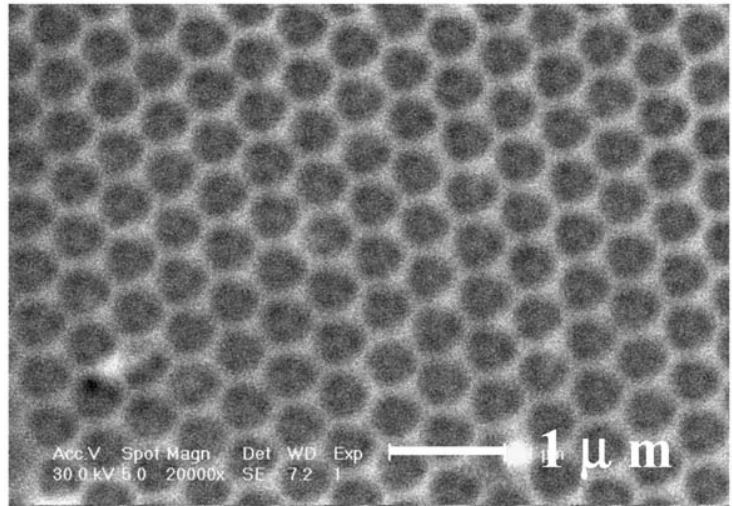

Fig. 1. SEM image of polypyrrole macroporous template film.

amount of charge passed during electrodeposition. For small values of $t$ these structures form ordered arrays of disconnected dots but if the amount of material deposited is increased these dots join up forming ordered magnetic networks. In the former case the dots have a near-spherical or "droplet" shape with a bottom part reproducing the spherical shape of the template cavity and a top part, which is dome-shaped due to the growth process. The dot array has a period $d$, which is equal to the diameter of the polystyrene spheres used in template preparation. The morphology of the films and their nano-structure were studied using electron microscopy. Fig. 2 shows an SEM image of connected and isolated $\mathrm{Ni}$ dot arrays prepared by the double template deposition method. Fig. 2b also demonstrates the "droplet" shape of dots. Magnetic measurements were performed at room temperature using a vibrating sample magnetometer and a SQUID magnetometer.

\section{Results and discussion}

The magnetic properties of Ni nano-dot arrays produced by the above-described method have been studied. In particular, we investigated arrays of isolated dots with a ratio $t / d=\frac{1}{3}$ prepared from spheres of diameters $d$ in a range from 200 to $700 \mathrm{~nm}$. It is known that for lithographically prepared nano-scale dots in a particular size range the most favorable state is a vortex state [8-10]: the 


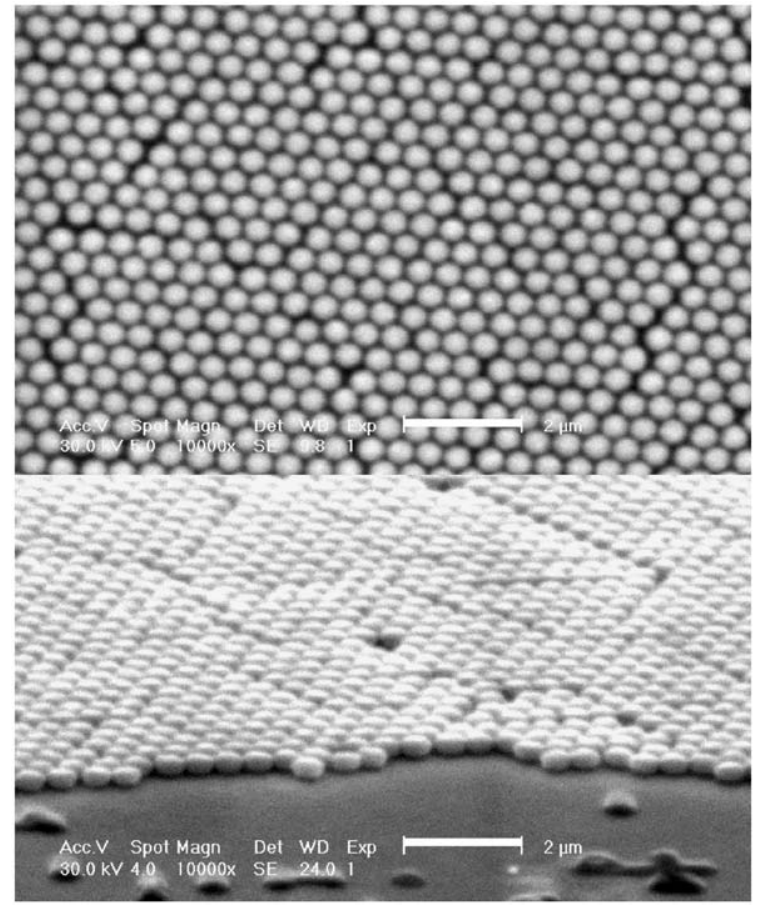

Fig. 2. SEM image of isolated (a) and interconnected (b) Ni dot arrays prepared using polystyrene spheres of $500 \mathrm{~nm}$ diameter.

magneto-static energy can be lowered by introducing a circular configuration in the distribution of magnetic moments. In the case of large particles this decrease in magnetic energy dominates the increase of exchange energy but in small dots the formation of vortices costs too much in exchange energy and a single-domain state becomes the most stable state. The critical size for the transition between vortex and single-domain behavior will be affected by dot shape, inter-dot magnetic dipolar interactions and the magneto-crystalline anisotropy of the dots. We have found that for unpatterned $\mathrm{Ni}$ films produced by the same electrochemical method, the behavior is magnetically soft. In addition, in the arrays investigated, the dots are well-separated. Hence the magnetic properties are dominated by exchange interactions and intra-dot magnetostatic energies. Our measurements on the dot arrays allow us to study how magnetization reversal mechanism depends on dot size and at which size the cross-over between vortex and single-domain state occurs in dots with a quasi-spherical shape. For large dots with $d>200 \mathrm{~nm}$ the magnetic measurements, performed using the VSM, showed nearly reversible magnetization curves. Our findings suggest that for $d$ between 200 and $700 \mathrm{~nm}$ the dots undergo vortexlike magnetization processes [11]. In Fig. 3, we present magnetization curves for dots with $d=350 \mathrm{~nm}$ (a) and $500 \mathrm{~nm}$ (b) measured with the magnetic field applied in the plane of the array film. The magnetization reaches saturation in magnetic fields $\sim 1 \mathrm{kOe}$ and demonstrates reversible behavior within the resolution of our magnetometer. But in arrays of smaller dots the magnetic behavior changes. Magnetization curves for a dot array with $d=200 \mathrm{~nm}$ are shown in Fig. 4. It can be seen that for the magnetic field applied in the plane of the array the magnetization reversal is similar to a single-domain behavior-relatively large hysteresis with a value of the coercive field of about 720 Oe. The squareness of the loop

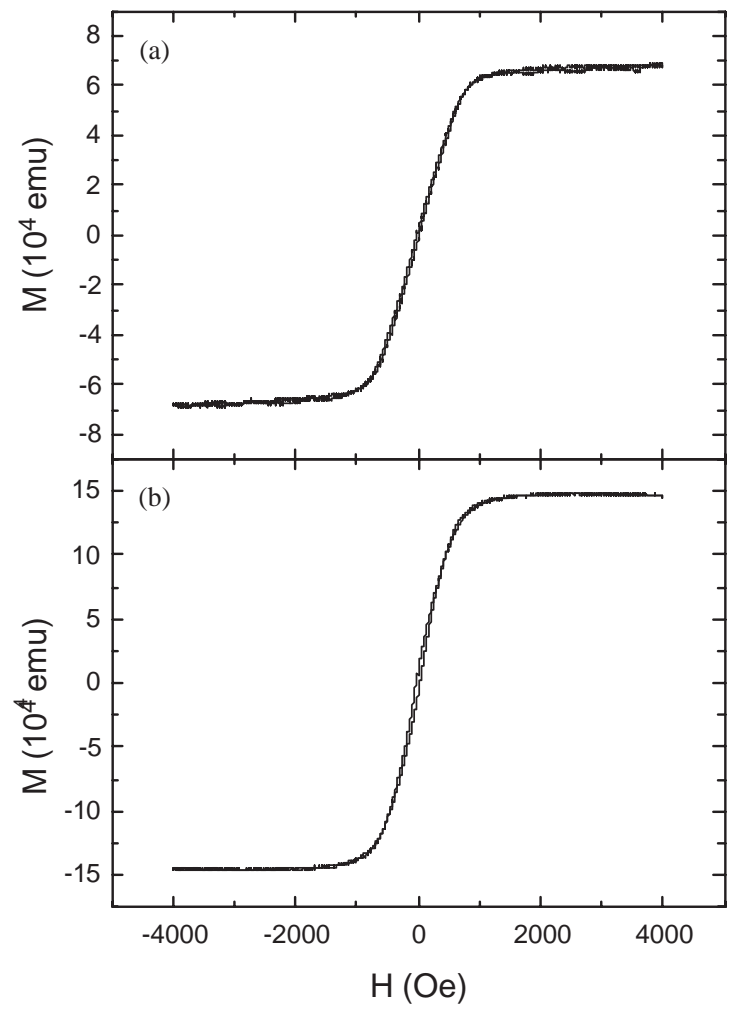

Fig. 3. Magnetization loops, measured by VSM, for an array of $\mathrm{Ni}$ dots prepared from $350 \mathrm{~nm}$ (a) and $500 \mathrm{~nm}$ (b) spheres. 


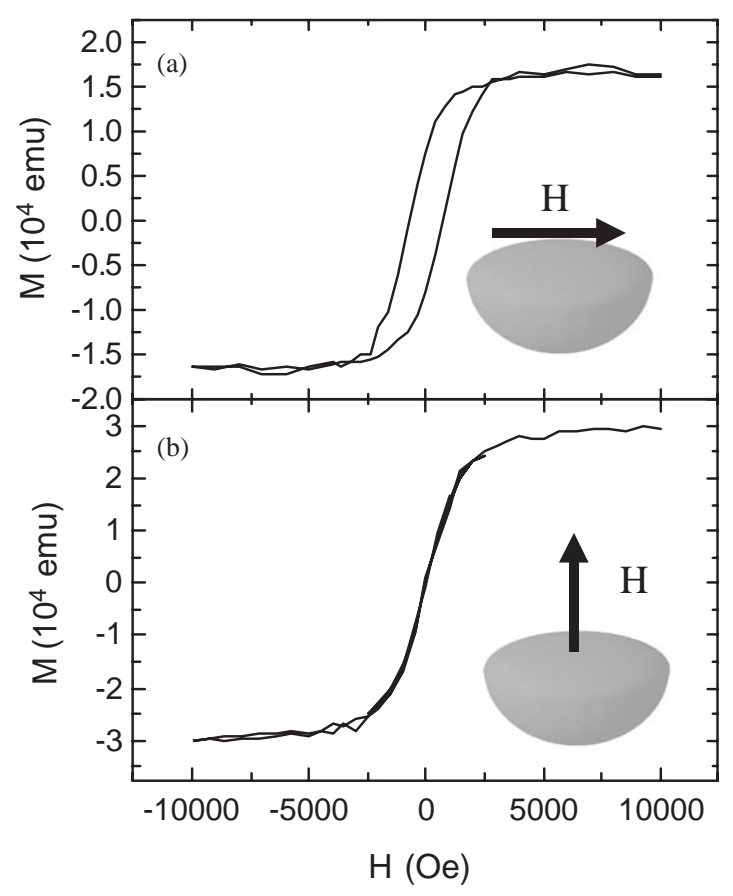

Fig. 4. Magnetization curves for the field applied parallel to the array plane (a) and transverse to the plane (b) of $\mathrm{Ni}$ dots prepared using a template of $200 \mathrm{~nm}$ spheres.

$M_{\text {rem }} / M_{\text {sat }}$ is 0.45 . For lithographically prepared nano-discs of thickness 10 and $15 \mathrm{~nm}$ the transition between vortex and single-domain behavior takes place for somewhat smaller diameters: $150-200 \mathrm{~nm}$ [10]. This disagreement may be caused by the difference in materials of the discs and our dots, dot shape and thickness, which in our case was $\sim 70 \mathrm{~nm}$. In contrast to the case for fields in the array plane, the behavior for magnetic fields applied transverse to the array reveals a magnetization curve, which remains reversible. We interpret this in terms of the dot shape, which has an aspect ratio smaller than one: the dot diameter is larger than its thickness. For fields in the array plane it is energetically unfavorable for a vortex to form. Due to the smaller length scale over which the magnetization would have to turn, the increase in exchange energy inhibits the formation of a vortex. For magnetic fields perpendicular to the array plane, the vortex is set up in the circular cross-section of the dot, which has a lower cost in exchange energy. These findings were found to be in agreement with results of 3-D micromagnetic calculations for small dots of the same shape [12]; giving values for the critical size of about $140 \mathrm{~nm}$. The somewhat smaller critical value of found $d$ in the simulations is probably related to the uncertainty in the value of the exchange parameter for Ni. Several other factors like details of dot shape and interactions between dots may also contribute to this difference.

\section{Conclusion}

We have described a novel method for the fabrication of highly ordered arrays of magnetic dots. Results of magnetization measurements show that the magnetic properties change depending on nano-structuring. We have found evidence for the existence of vortex and single-domain states in small dots with quasi-spherical shape depending on dot size and field direction.

\section{Acknowledegments}

This work has been supported by the EPSRC (Grant GR/29396). A.G. also acknowledges the support of the Institute of Physics Magnetism group and the $\mathrm{C}$ R Barber Trust.

\section{References}

[1] M. Helm, et al., Science 272 (1996) 1782.

[2] R.P. Cowburn, J. Magn. Magn. Mater 242-245 (2002) 505.

[3] J.I. Martin, et al., J. Magn. Magn. Mat. 256 (2003) 449-501.

[4] R. Zhu, et al., Chem. Phys. Lett. 368 (2003) 696-700.

[5] Mikrajuddin, et al., Adv. Mat. 14 (2002) 930-933.

[6] M.S. Sander, et al., Adv. Func. Mat. 13 (2003) 393-397.

[7] M.A. Ghanem, et al., J. Mat. Chem. 11 (2001) 849.

[8] T. Shinjo, et al., Science 289 (2000) 930.

[9] A. Lebib, et al., J. Appl. Phys 89 (2001) 3892.

[10] R.P. Cowburn, et al., Phys. Rev. Lett. 83 (1999) 1042-1045.

[11] A. A. Zhukov, et al., J. Magn. Magn. Mater, in print.

[12] R. Boardman, et al., J. Appl. Phys., in print. 